\title{
Period-tripling subharmonic oscillations in a driven superconducting resonator
}

\author{
Ida-Maria Svensson, ${ }^{*}$ Andreas Bengtsson, Philip Krantz, Jonas Bylander, Vitaly Shumeiko, and Per Delsing ${ }^{\dagger}$ \\ Microtechnology and Nanoscience, Chalmers University of Technology, SE-41296 Göteborg, Sweden \\ (Received 13 July 2017; revised manuscript received 18 October 2017; published 8 November 2017)
}

\begin{abstract}
We have observed period-tripling subharmonic oscillations in a driven superconducting coplanar waveguide resonator operated in the quantum regime, $k_{B} T \ll \hbar \omega$. The resonator is terminated by a tunable inductance that provides a Kerr-type nonlinearity. We detected the output field quadratures at frequencies near the fundamental mode, $\omega / 2 \pi \sim 5 \mathrm{GHz}$, when driving the resonator with a current at $3 \omega$, with amplitude exceeding an instability threshold. We observed three stable radiative states with equal amplitudes, phase shifted by $2 \pi / 3$ rad, red detuned from the fundamental mode. The down-conversion from $3 \omega$ to $\omega$ is strongly enhanced by near-resonant excitation of the second mode of the resonator and the cross-Kerr effect. Our experimental results are in quantitative agreement with a model for the driven dynamics of two coupled modes.
\end{abstract}

DOI: 10.1103/PhysRevB.96.174503

\section{INTRODUCTION}

Nonlinear dynamical systems exhibit a vast variety of behaviors, from simple effects such as harmonic generation to sophisticated multiple bifurcations to pattern formation and chaos [1-3]. Particularly interesting are strongly nonlinear phenomena in the quantum regime, which can be realized in low-dissipation microwave systems such as circuit quantum electrodynamics (cQED) devices. Such phenomena play a central role and are widely employed in quantum information technology for qubit readout [4-6], photon entanglement [7-10], and generation of Schrödinger cat states [11,12].

Period-multiplying subharmonic oscillations [13,14] constitute a particular class of nonlinear phenomena. The oscillations appear as a nonlinear response at the oscillator frequency to an external drive at a multiple of the resonant frequency. In the quantum picture, the elementary process that underlies the subharmonic oscillations is a decay of a single photon into three, four, or more photons. The subharmonic oscillations are described by nonperturbative solutions to the dynamical equations, which appear abruptly and coexist with the stable vacuum state. In this respect the subharmonic oscillations distinctly differ from conventional parametric oscillations, which gradually emerge as a result of the vacuum instability. This difference is analogous to the difference between a first- and a second-order phase transition [15]. Furthermore, a symmetry-breaking aspect of this difference has important implications for the quantum dynamics of the period-tripling oscillations [16].

Although the period-multiplying phenomenon is theoretically explained in textbooks, experimental demonstrations are not common. A few early observations of subharmonic resonances in electromagnetic devices were performed on essentially classical electrical circuits with saturable inductors [17] or varactors [18]. More recent reports concern subharmonic resonances in lasers [19,20]. In Josephson circuits, the period-multiplying phenomenon has not received much attention; instead, research was focused on transition to chaos $[21,22]$ and, lately, on bifurcation phenomena [23,24] and

\footnotetext{
*ida-maria.svensson@ chalmers.se

†per.delsing@chalmers.se
}

parametric oscillations [5,6,25-27]. Only recently have subharmonic oscillations in the quantum regime been theoretically discussed in the context of cQED [16,28,29].

In this paper we report the experimental observation of period-tripling subharmonic oscillations in a driven superconducting resonator in the quantum regime, $k_{B} T \ll \hbar \omega$ [30-32], where the thermal energy is much smaller than the energy of a single photon. We drive the nonlinear resonator with a harmonic signal with power $P_{d}$ at frequency $3 \omega$, approximately equal to three times the fundamental resonator mode frequency, and observe a strong response at $\omega$. The output microwave signal consists of three correlated beams with equal amplitudes and different phases, shifted by $2 \pi / 3$ rad. The oscillations are detected within a certain window of the driving field amplitude: they start at finite-threshold detuning within the resonator bandwidth and persist deep into the red-detuning region.

Our observations can be qualitatively understood from the theory of a nonlinear oscillator $[13,14]$. When a driving force with off-resonant frequency $3 \omega$ is applied, it generates a linear response at the same frequency, which is down-converted to frequency $\omega$ due to nonlinearity. The down-conversion has the highest efficiency when the detuning, $\delta_{1}=\omega-\omega_{1}$, from the oscillator resonant frequency $\omega_{1}$ is small, $\delta_{1} \ll \omega_{1}$.

However, application of this single-mode scenario to the resonator setting requires additional considerations. In our experiment, we drive the resonator close to its second mode, $\omega_{2}$, such that $\delta_{2}=3 \omega-\omega_{2} \ll \omega_{1}$. As a result, the response at the driving frequency becomes strongly enhanced and nonlinear, and the system dynamics is well described by two strongly interacting modes. This situation is different from the single-mode oscillator model explored in previous works on subharmonic oscillations. In fact, this resonant enhancement of the external drive, by more than three orders of magnitude, is crucial for the possibility to access the subharmonic oscillation regime in experiments.

\section{EXPERIMENT}

To observe subharmonic oscillations we use frequencytunable coplanar waveguide microwave resonators [30-32]. The resonator is capacitively coupled to a $50 \Omega$ transmission line on one end and grounded via a superconducting quantum 

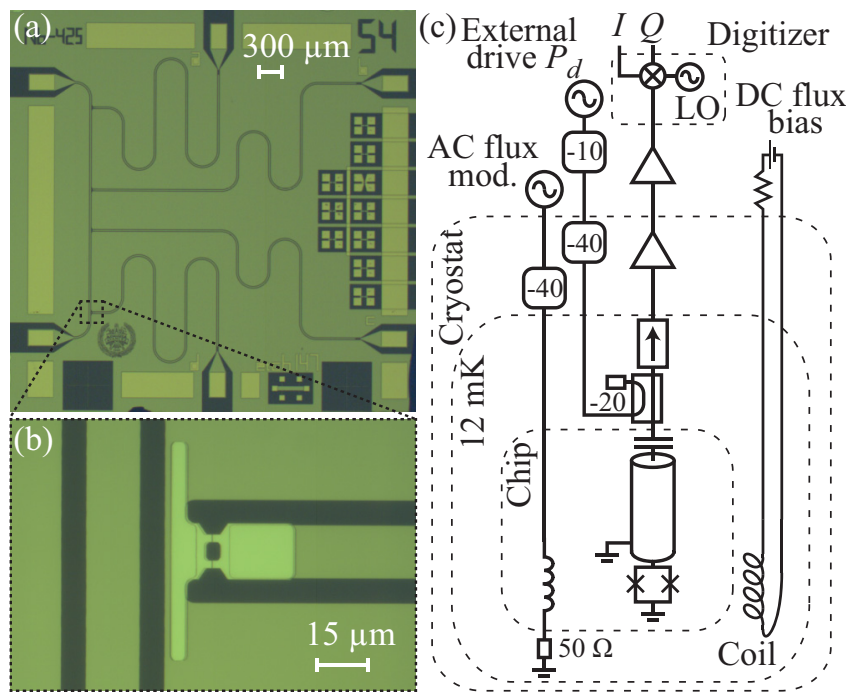

FIG. 1. (a) Optical micrograph of one of the samples. The four coplanar waveguide resonators meander between the on-chip flux line on the left and the contact pads on the right. (b) Zoom of the SQUID that terminates the bottom resonator. The SQUID is designed with two identical Josephson junctions. (c) Measurement setup. Microwave signals are applied via attenuated coaxial cables, one for direct driving by an external ac current and one for flux modulation. To separate input and output, a directional coupler is used to route the signal. A static magnetic flux is induced by a superconducting coil. The output signal is amplified in a 4-8-GHz bandwidth by a cryogenic amplifier as well as a room-temperature amplifier. The quadrature voltages are acquired by heterodyne detection followed by digital demodulation.

interference device (SQUID) on the other end (see Fig. 1). In practice, the SQUID acts as a tunable nonlinear inductance controlled by the magnetic flux $\Phi$ threading its loop and the current $I_{s}, L_{J}\left(\Phi, I_{s}\right)=\Phi_{0} /\left[2 \pi\left|\cos \left(\pi \Phi / \Phi_{0}\right)\right| \sqrt{I_{c}^{2}-I_{s}^{2}}\right]$, where $\Phi_{0}=h / 2 e$ is the magnetic flux quantum and $I_{s}$ and $I_{c}$ denote the current flowing through the device and its critical current, respectively. The Josephson nonlinearity of the SQUID induces, at weak excitation, a Duffing-Kerr nonlinearity in the resonator.

The samples are fabricated using standard processes: Josephson junctions are deposited by two-angle evaporation of aluminum. The rest of the circuit is etched out of a sputtered layer of niobium on a sapphire wafer. A micrograph of one chip with four resonators of different lengths is shown in Fig. 1(a). The chip is anchored to the mixing chamber stage of a dilution refrigerator with a base temperature of $12 \mathrm{mK}$.

The measurement setup is sketched in Fig. 1(c). For static magnetic flux biasing of the SQUID we use a superconducting coil mounted close to the sample box. The SQUID nonlinearity can be modulated by applying a microwave signal as an external drive of the current through the SQUID $I_{s}$ or by flux modulation. We focus mainly on the external driving. The resonator output signal is amplified by a low-noise cryogenic amplifier at the $3 \mathrm{~K}$ stage, as well as an additional amplifier at room temperature, before being sampled by a digitizer. To maintain phase coherence, a $10-\mathrm{MHz}$ signal is used to lock the signal generator and the digitizer together. The digitizer down-converts both the in- and out-of-phase quadratures, $I(t)$

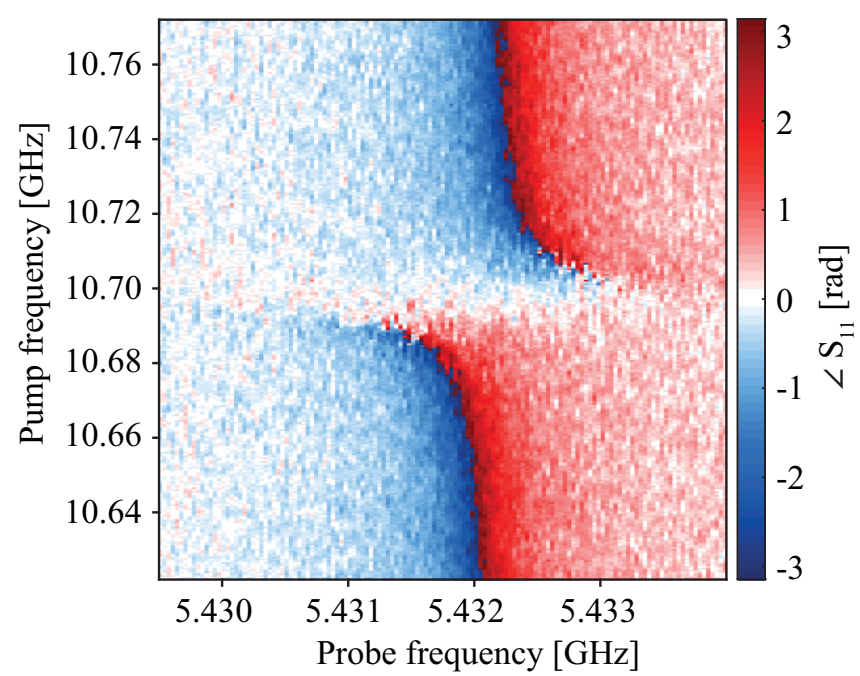

FIG. 2. Determination of the second mode frequency via parametric up-conversion. A weak probe tone (horizontal axis), near the resonant frequency of the first mode $\omega_{1}$, is up-converted by the flux pump tone (vertical axis), near the difference frequency $\omega_{2}-\omega_{1}$. The process results in an avoided level crossing visible in the reflected probe tone. The data presented show the reflected phase response for a magnetic flux bias, $\Phi=0.17 \Phi_{0}$, where $\omega_{1} / 2 \pi=5.432 \mathrm{GHz}$ and $\left(\omega_{2}-\omega_{1}\right) / 2 \pi=10.70 \mathrm{GHz}$, yielding $\omega_{2} / 2 \pi=16.132 \mathrm{GHz}$.

and $Q(t)$, with a local oscillator before digitizing the data at an effective sampling rate $f_{s}$ during a time $t_{s}$. From the individual quadratures the total output power after amplification can be calculated as $P_{\text {out }}=\left\langle I^{2}\right\rangle+\left\langle Q^{2}\right\rangle$.

We directly probe the first resonator mode in a reflection measurement. Higher modes can be measured only indirectly due to the $4-8-\mathrm{GHz}$ bandwidth limitation of our setup. To detect the second mode we use parametric up-conversion [33,34]: we modulate the magnetic flux penetrating the SQUID loop at the difference frequency of the first and the second modes, $\omega_{2}-\omega_{1}$, while simultaneously applying a weak drive tone at $\omega_{1}$. The flux pump converts photons from the first to the second mode, resulting in an avoided crossing, as shown in Fig. 2, from which we can determine the difference frequency.

In Fig. 3(a) we present the extracted frequencies of the lowest mode for our sample together with a fit to the spectral dispersion, Eq. (A1). The extracted parameters are presented in Table I. The resonator is overcoupled and has a narrow bandwidth, less than $1 \mathrm{MHz}$.

\section{SUBHARMONIC OSCILLATIONS}

\section{A. Observations}

We observe period-tripling subharmonic oscillations at $\omega \approx \omega_{1}$ by applying an external signal at $3 \omega$. This effect has been observed in several samples, although in this paper we present data for only one sample. As can be seen in Fig. 4(a), we observe subharmonic oscillations in a range of red detuning, $\delta_{1}=\omega-\omega_{1}<0$, and for signal generator drive powers above $\sim-13 \mathrm{dBm}$. This range of drive powers and detunings forms region II, where the subharmonic oscillations are visible. Above and below, in regions I and III, respectively, no subharmonic oscillations are observed. 
(a)

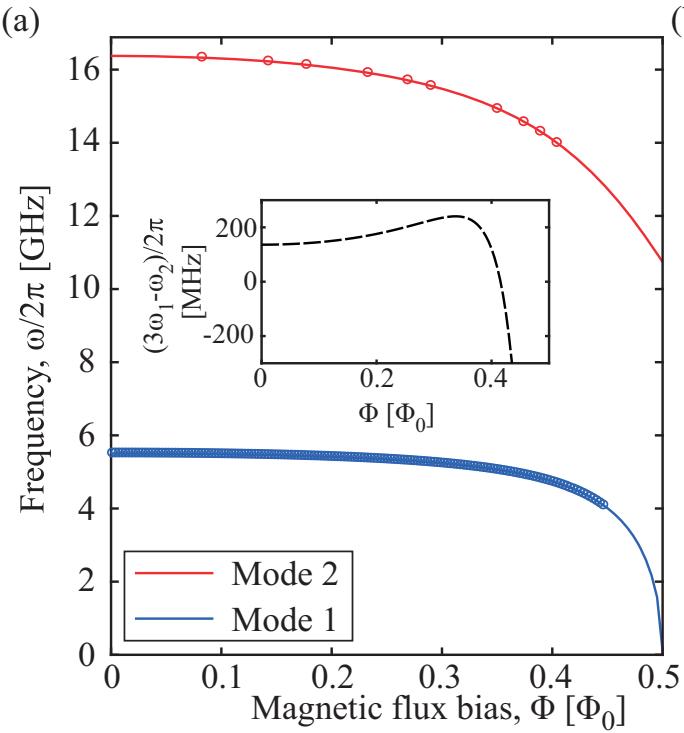

FIG. 3. (a) Spectroscopy of resonator modes 1 and 2, fitted using Eq. (A1). Extracted resonator and SQUID parameters are found in Table I. Inset: anharmonicity of the spectrum, $3 \omega_{1}-\omega_{2}$. (b) An illustration of the frequency spectrum. Since the spectrum is nonequidistant, the drive signal around $3 \omega$ falls slightly above mode 2 , while the measurement frequency $\omega$ is slightly below mode 1 .

We also investigated the quadratures of the oscillations using histograms of the $I(t)$ and $Q(t)$ signals. In Fig. 4(c) we show a background histogram (peaked at $I=Q=0$ ) illustrating the system noise level. This histogram represents the system ground state. At higher drive power, inside region II, the histograms feature three well-defined stable states forming a regular triangle [see Figs. 4(e) and 4(f)]. At the low-power edge of region II the system shows four states [see Fig. 4(d)]. This observation is in full agreement with the phase portrait of the subharmonic oscillator (see Fig. 7) featuring four coexisting stable steady states: the silent ground state and the three excited states.

The system switching rates between the states are different for different operating points, $\delta_{1}$ and $P_{d}$. Analysis of the underlying time-domain data yields a switching rate of $1 \mathrm{kHz}$ in Fig. 4(d) and $15 \mathrm{kHz}$ in Fig. 4(f). In Figs. 4(d) and 4(e) the histograms show clearly separated states, while in Fig. 4(f) the states are connected by faint lines. These lines indicate enhancement of the stochastic switching between the steady states. When the system switching rate becomes comparable to the sampling rate, $f_{s w} \sim f_{s}$, the states are averaged together.

Lowering the sampling rate to $10 \mathrm{kHz}$ makes the switching processes between the stationary states more visible (see
Fig. 5). In region II the transitions occur only between the excited states, forming a triangle configuration, while at the border of regions II and III the transitions connect the ground and excited states, forming a star configuration.

\section{B. Theory}

To explain the experimental observations and establish a basis for quantitative comparison, we perform a theoretical analysis based on the theory for two-mode resonant dynamics in a frequency-tunable resonator [26,34]. The two-mode equations for slowly varying Heisenberg operators of the coupled modes, $a_{1}$ and $a_{2}$, in the doubly rotating frame with frequencies $\omega$ and $3 \omega$ have the form

$$
\begin{aligned}
& i \dot{a}_{1}+\left(\delta_{1}+i \Gamma_{1}+\alpha_{1} a_{1}^{\dagger} a_{1}+2 \alpha a_{2}^{\dagger} a_{2}\right) a_{1}+\tilde{\alpha} a_{1}^{\dagger 2} a_{2}=0, \\
& i \dot{a}_{2}+\left(\delta_{2}+i \Gamma_{2}+\alpha_{2} a_{2}^{\dagger} a_{2}+2 \alpha a_{1}^{\dagger} a_{1}\right) a_{2}+\frac{\tilde{\alpha}}{3} a_{1}^{3} \\
& \quad=\sqrt{2 \Gamma_{2, \mathrm{ext}}} B_{2} .
\end{aligned}
$$

Here, the amplitude of the fundamental mode, $a_{1}$, describes the subharmonic oscillator, while that of the second mode, $a_{2}$, acts as an effective parametric pump. $B_{2}$ is the complex amplitude of the external drive and $\Gamma_{n}$ is the mode damping. Explicit equations for the external damping $\Gamma_{2, \mathrm{ext}}$ and the Kerr coefficients $\alpha_{n}$ are presented in Eqs. (A5) and (A6). The cross-Kerr coefficients are related to the Kerr coefficients, $\alpha=\sqrt{\alpha_{1} \alpha_{2}}$ and $\tilde{\alpha}=4 / \alpha_{1}^{3} \alpha_{2}$. Equations (1) are associated with and can be derive from the quantum Hamiltonian,

$$
\begin{aligned}
H / \hbar= & -\sum_{n=1,2}\left(\delta_{n} a_{n}^{\dagger} a_{n}+\frac{\alpha_{n}}{2} a_{n}^{\dagger 2} a_{n}^{2}\right)-2 \alpha a_{1}^{\dagger} a_{1} a_{2}^{\dagger} a_{2} \\
& -\frac{\tilde{\alpha}}{3}\left(a_{1}^{\dagger 3} a_{2}+a_{1}^{3} a_{2}^{\dagger}\right)+\sqrt{2 \Gamma_{2, \mathrm{ext}}}\left(B_{2} a_{2}^{\dagger}+B_{2}^{*} a_{2}\right) .
\end{aligned}
$$

Subharmonic oscillations are essentially a classical phenomenon since a large number of photons is generated in the resonator. Thus we restrict the analysis to the quasiclassical solutions of Eq. (1), neglecting quantum effects. A detailed description of the eigenfunctions and tunneling rates can be found in Ref. [16] for the Hamiltonian (2) in the single-mode case.

The trivial quasiclassical solution to Eq. (1), $a_{1}=0$, describes a silent oscillator state. It is always stable [see the Appendix, Eq. (A3)]. The nontrivial solutions describing stable steady states of the excited oscillator consist of a phasedegenerate triad, with the states being stable within the region of existence, Eq. (A14). In terms of a polar parametrization of

TABLE I. Resonator and SQUID parameters: $d$, resonator length; $I_{c}$, SQUID critical current; $C_{J}$, SQUID capacitance; $\gamma_{0}=L_{J}^{0} / L_{0} d$, the inductive participation ratio; $L_{0}$ and $C_{0}$, inductance and capacitance per unit length; $\omega_{1}(0)$, resonant frequency of the first mode at $\Phi=0$; $3 \omega_{1}(0)-\omega_{2}(0)$, spectrum anharmonicity; $2 \Gamma_{1}(0)$, damping of the first resonator mode; $Q_{1, \text { int }}$ and $Q_{1 \text {,ext }}$, quality factors of the fundamental mode.

\begin{tabular}{lcccccccccc}
\hline \hline $\begin{array}{l}d \\
(\mu \mathrm{m})\end{array}$ & $\begin{array}{c}I_{c} \\
(\mu \mathrm{A})\end{array}$ & $\begin{array}{c}C_{J} \\
(\mathrm{fF})\end{array}$ & $\begin{array}{c}\gamma_{0} \\
(\%)\end{array}$ & $\begin{array}{c}L_{0} \\
(\mu \mathrm{H} / \mathrm{m})\end{array}$ & $\begin{array}{c}C_{0} \\
(\mathrm{nF} / \mathrm{m})\end{array}$ & $\begin{array}{c}\omega_{1}(0) / 2 \pi \\
(\mathrm{GHz})\end{array}$ & $\begin{array}{c}{\left[3 \omega_{1}(0)-\omega_{2}(0)\right] / 2 \pi} \\
(\mathrm{MHz})\end{array}$ & $\begin{array}{c}2 \Gamma_{1}(0) / 2 \pi \\
(\mathrm{MHz})\end{array}$ & $Q_{1, \mathrm{int}}(0)$ & $Q_{1, \mathrm{ext}}(0)$ \\
\hline 5080 & 1.90 & 86.1 & 7.7 & 0.44 & 0.16 & 5.504 & 136 & 0.38 & $61.1 \times 10^{3}$ & $19 \times 10^{3}$ \\
\hline \hline
\end{tabular}


(a)

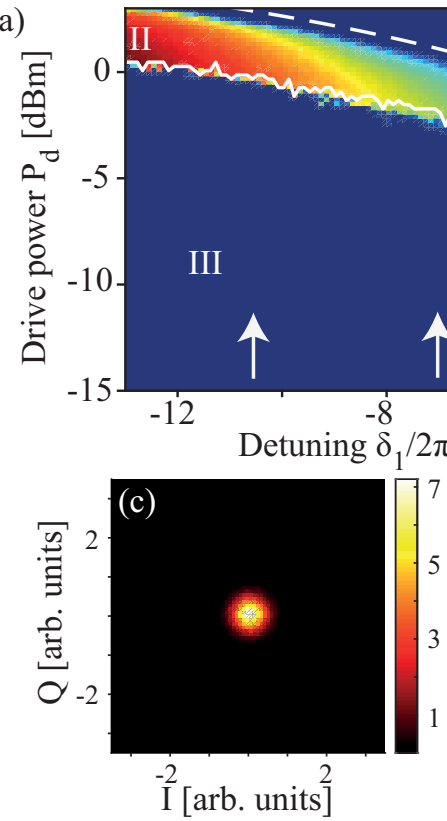

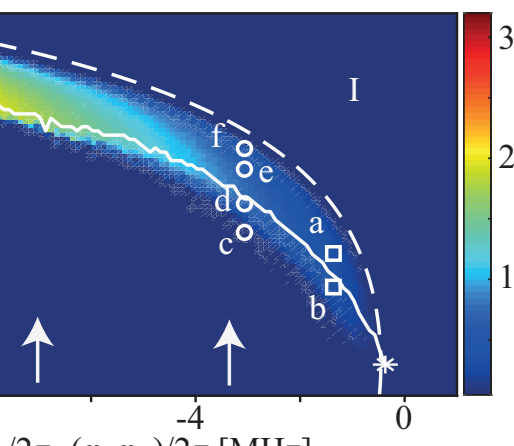

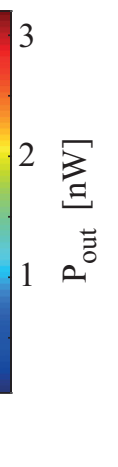

(b)
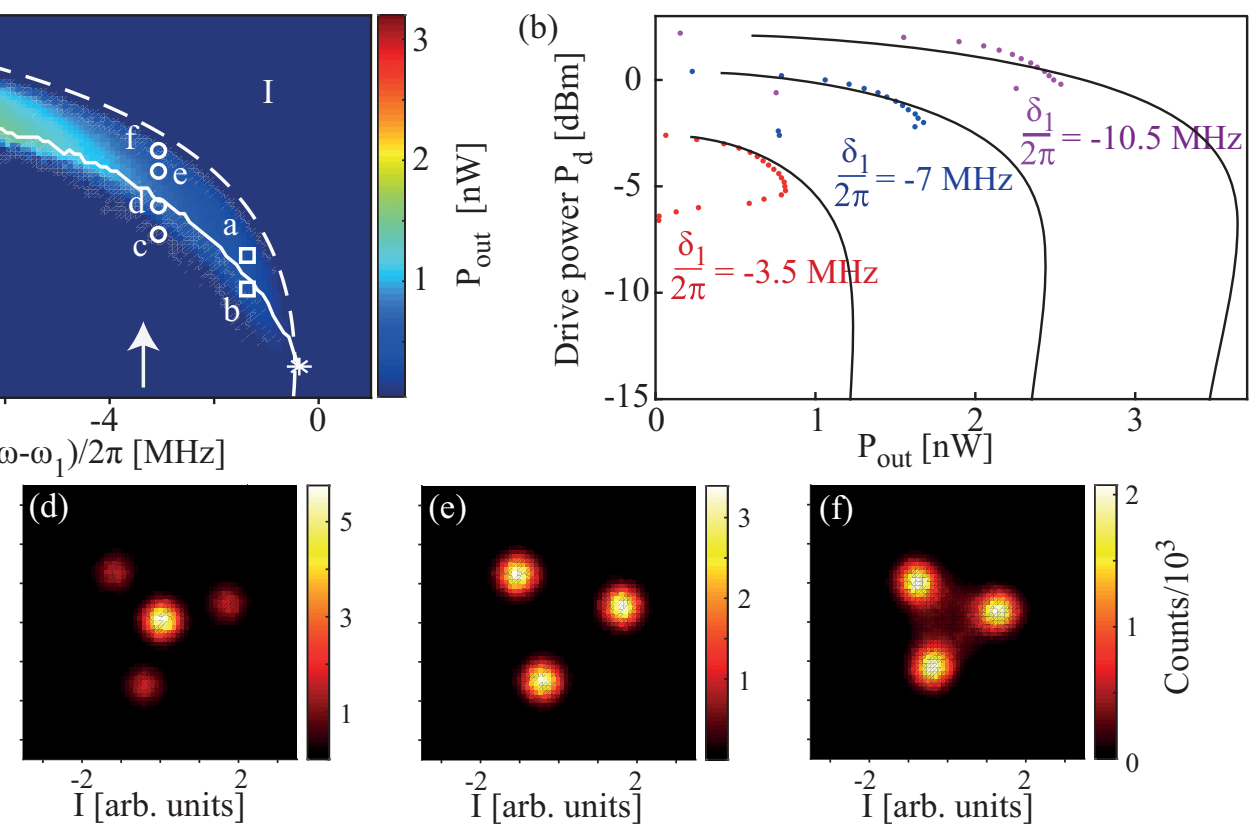

FIG. 4. (a) Intensity of the subharmonic oscillation output signal as a function of drive power and detuning for $\Phi=0$. The oscillations are detected in region II. The solid white line corresponds to the maximum output signal for each measurement frequency; the white star at the end of the solid white line represents the subharmonic oscillation frequency threshold. The dashed white line that separates regions I and II corresponds to the theoretical boundary of existence for the subharmonic oscillations. In region III, the oscillations, although they exist as a solution to Eqs. (1), are not visible because the oscillator switches to the ground state. (b) The dots represent three linecuts from (a) at detuning values indicated by the white arrows; a theory fit to these three data traces is represented by the black lines. (c)-(f) Histograms of the detected radiation quadrature voltages from the operating points indicated by the white circles in (a). These histograms reveal three dynamic states: (c) the ground state, i.e., the background noise level; (d) the ground state (in the middle) and the three excited states with equal amplitudes and with phases differing by $2 \pi / 3$; and (e) and (f) the three excited states. All histograms are sampled at a rate of $f_{s}=100 \mathrm{kHz}$. The features in (f) are connected by faint lines, an artifact from the averaging that indicates that the switching rate at this point is higher than in (d) and (e) and not negligible compared to $f_{s}$. These histograms can be compared to the histograms sampled with $f_{s}=10 \mathrm{kHz}$ that are presented in Fig. 5 and were measured with parameters indicated by the white squares in (a).

the quasiclassical field amplitudes,

$$
a_{1}=r_{1} e^{i \phi_{1}}, \quad a_{2}=\frac{r_{2}}{\beta} e^{i \phi_{2}}, \quad \theta=3 \phi_{1}-\phi_{2},
$$

with $\beta=\sqrt[4]{\alpha_{2} / \alpha_{1}}$, the stable solution has the form

$$
\begin{aligned}
r_{1}^{2} & =\frac{\left|\delta_{1}\right|}{\alpha_{1}}-\frac{3 r_{2}^{2}}{2}+\sqrt{\frac{r_{2}^{2}\left|\delta_{1}\right|}{\alpha_{1}}-\frac{7 r_{2}^{4}}{4}-\frac{\Gamma_{1}^{2}}{\alpha_{1}^{2}},} \\
\sin \theta & =\frac{\Gamma_{1}}{\alpha_{1} r_{2} r_{1}}, \quad \theta \in(\pi / 2, \pi) \bmod (2 \pi) .
\end{aligned}
$$

The solution (4) exists within an interval of the effective pump intensity $r_{2 \pm}^{2}$,

$$
r_{2}^{2} \in\left(r_{2-}^{2}, r_{2+}^{2}\right), \quad r_{2 \pm}^{2}=\frac{2\left|\delta_{1}\right|}{7 \alpha_{1}}\left[1 \pm \sqrt{1-\frac{7 \Gamma_{1}^{2}}{\delta_{1}^{2}}}\right],
$$

and at negative red detuning from the fundamental resonator mode,

$$
\delta_{1} \leqslant-\sqrt{7} \Gamma_{1}
$$

The solution (4) is finite at the boundaries of existence (6); that is, the subharmonic oscillations emerge abruptly when the boundaries are crossed. The oscillations achieve a maximum intensity,

$$
r_{1, \max }^{2}=\frac{4}{7 \alpha_{1}}\left(\left|\delta_{1}\right|+\sqrt{\left|\delta_{1}\right|^{2}-7 \Gamma_{1}^{2}}\right),
$$

that grows linearly with the detuning far from the threshold, $\left|\delta_{1}\right| \gg \Gamma_{1}$. The maximum is achieved in this region at $r_{2}^{2}=$ $\left|\delta_{1}\right| / 14 \alpha_{1}$.

The effective pump strength $r_{2}$ is defined by a nonlinear response to the external drive $B_{2}$, Eq. (A8). The response exhibits instability at a weak drive, $\left|B_{2}\right|^{2} \lesssim \beta^{2}\left|\delta_{1}\right|^{3} /\left(18 \Gamma_{2, \text { ext }} \alpha_{1}\right)$, as shown in Figs. 8 and 9, but has a regular monostable behavior at larger drive, up to the maximum value given by Eq. (A18),

$$
\left|B_{2, \max }\right|^{2} \approx \frac{\left(3 \omega_{1}-\omega_{2}\right)^{2}\left|\delta_{1}\right|}{7 \beta^{2} \Gamma_{2, \mathrm{ext}} \alpha_{1}}, \delta_{1} \gg \Gamma_{1}, \Phi<0.4 \Phi_{0}
$$

The phase $\phi_{2}$ is defined by the phase of the drive $\phi_{B}$, and for the stable branch and large detuning, $\delta_{1} \gg \Gamma_{2}$, it is approximately $\pi$ shifted from the latter (see the Appendix). This situation persists within a wide interval of magnetic flux bias, $0<\Phi<0.4 \Phi_{0}$, as long as the anharmonicity of the resonator spectrum exceeds the detuning, $3 \omega_{1}-\omega_{2} \gg \delta_{1}$ (see the inset in Fig. 3). 


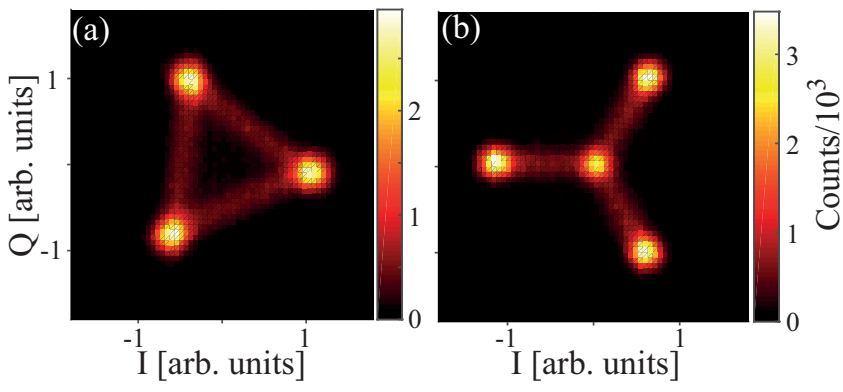

FIG. 5. Histograms sampled with a rate $f_{s}=10 \mathrm{kHz}$, ten times slower than in Figs. 4(c)-4(f). Here, the lines between the steady states are clearly seen. (a) The histogram measured well inside region II reveals a triangle configuration of transitions between the excitedstate features. (b) The histogram measured at the border between regions II and III exhibits a "star" configuration of transitions between the ground and excited states. These histograms are measured at $\delta_{1} / 2 \pi=1.5 \mathrm{MHz}$ and drive power as indicated by the white squares in Fig. 4(a).

\section{Analysis}

Using the outlined theoretical results, we are able to quantitatively analyze the details in Fig. 4. Figure 4(b) displays three linecuts of the subharmonic oscillation region taken at three different values of detuning. The oscillation amplitudes, represented by dots, show sharp onsets at the low-power edge of region II and smoother decays towards the high-power edge. The solid lines correspond to a theoretical fit. The power of the external drive is in linear units,

$$
P_{d}=3 \hbar \omega\left|B_{2}\right|^{2} 10^{A t t / 10},
$$

where Att is the attenuation (in $\mathrm{dB}$ ) between the generator and the resonator for the $3 \omega$ drive signal and $\left|B_{2}\right|^{2}$ is given by Eq. (A17). The measured output power is

$$
P_{\text {out }}=\hbar \omega\left|a_{1}\right|^{2} 2 \Gamma_{1, \mathrm{ext}} 10^{G / 10} .
$$

Here, $G$ denotes the overall gain of the measurement signal $\omega$ between the resonator and digitizer. The relation between the amplitudes of modes 1 and 2 is given in Eq. (4). The fit is done by adjusting only one fitting parameter, $X=Q_{2, \text { ext }} 10^{A t t} / 10$, which is found in Eqs. (10) and (A17), where $Q_{2 \text {,ext }}=\omega_{2} / 2 \Gamma_{2 \text {,ext }}$. The other parameters are measured independently and are listed in Table I; the Kerr coefficient $\alpha_{1} / 2 \pi=85 \mathrm{kHz}$ assumes the theory value, and the gain is estimated to be $G=66 \pm 0.5 \mathrm{~dB}$. The best fit is achieved for $X=(9.97 \pm 0.03) \times 10^{11}$. From this we can calculate the photon population of the first resonator mode $\left|a_{1}\right|^{2}$. It is found that $2.4 \mathrm{nW}$ of output power in Fig. 4(a) correspond to roughly 100 photons.

As seen in Fig. 4(b), the observed oscillations reach a maximum and disappear (the oscillator switches to the ground state) before they reach the theoretical maximum, Eq. (8). A comparison of the experimental and theoretical maxima reveals the scaling,

$$
\left|a_{1, \max }\right|_{\exp }^{2}=0.7\left|a_{1, \max }\right|^{2} .
$$

Using Eq. (A18) and fitting parameters extracted from Fig. 4(b), we evaluate the boundaries of existence and the stability of the subharmonic oscillations. The upper boundary is presented by the dashed white line in Fig. 4(a). Above the dashed line, in region I, the oscillations do not exist; below this line the theory predicts the existence of oscillations and stability within all of regions II and III [the oscillation lower boundary, Eq. (6), lies far below the edge of the panel]. However, the oscillations are visible only in the narrow region II but not in region III. This can be explained by a competition between the excited states and the stable ground state. At the boundary between regions II and III, the system explores all four available states, as indicated by Fig. 4(d), and in region III the system preferentially stays in the ground state [see Fig. 4(c)]. Quantitative evaluation of the lower boundary of visibility of the subharmonic oscillations requires a dynamical analysis including the effect of noise, which goes beyond the scope of the present study.

In Fig. 4 all data are taken at zero magnetic flux, $\Phi=0$. However, the subharmonic oscillations are detected also at nonzero flux up to $\Phi \approx 0.4 \Phi_{0}$. In Fig. 6(a) we present the maximum output power as a function of detuning for different flux bias values. At $\Phi=0$ this corresponds to the white solid line in Fig. 4(a). The output power is proportional to the maximum population of the first resonator mode and grows linearly with the detuning, in good agreement with the theory [Eq. (8)]. Furthermore, the flux dependence of the line slopes in Fig. 6(a) is in good agreement with the theory prediction given by the flux dependence of the Kerr coefficient (A6) in Eq. (8) and making use of the scaling, Eq. (12), as illustrated in Fig. 6(b).

The subharmonic oscillations are predicted to start at a threshold at small red detuning, Eq. (7). Experimentally, this threshold is defined as the end point of the white curve in Fig. 4(a), marked with a white star. Experimental data for the frequency thresholds at different $\Phi$ are presented in Fig. 6(c) (blue dots). For smaller flux values, $|\Phi| \lesssim 0.4 \Phi_{0}$, the threshold values of the output radiation, Eq. (11), evaluated for $\delta_{1}=-\sqrt{7} \Gamma_{1}$ exceed the noise level, $P_{n} \approx 0.44 \mathrm{nW}$, and therefore the measurement procedure identifies the true threshold, Eq. (7). However, at the edges of this region, $|\Phi| \approx 0.4 \Phi_{0}$, the output power rapidly decreases, as indicated in Figs. 4(a) and 4(b), and therefore the visible oscillation threshold shifts to larger detuning. Quantitatively, the shifted position of the threshold is defined by $P_{\text {out }}\left(\left|a_{1, \max }\right|^{2}\right)=$ $P_{n}$. We compute the solution to this equation using the parameters in Table I and the flux dependence of $\Gamma_{1, \text { ext }}$ in Eq. (A5). The internal losses and noise power are assumed to be flux independent. The theory plot of this solution, the red line in Fig. 6(c), excellently reproduces the data. We note that no fitting parameters were used in Figs. 6(b) and $6(\mathrm{c})$.

\section{CONCLUSION}

We observed period-tripling subharmonic oscillations in a driven nonlinear multimode microwave resonator in the quantum regime. When an external drive tone was applied at a frequency $3 \omega$, we observed output oscillations at $\omega$, demonstrating period tripling. The output signal consists of three correlated beams having the same amplitudes but with their phases shifted by $2 \pi / 3 \mathrm{rad}$ with respect to each other. The oscillations are observed at red detuning from the 

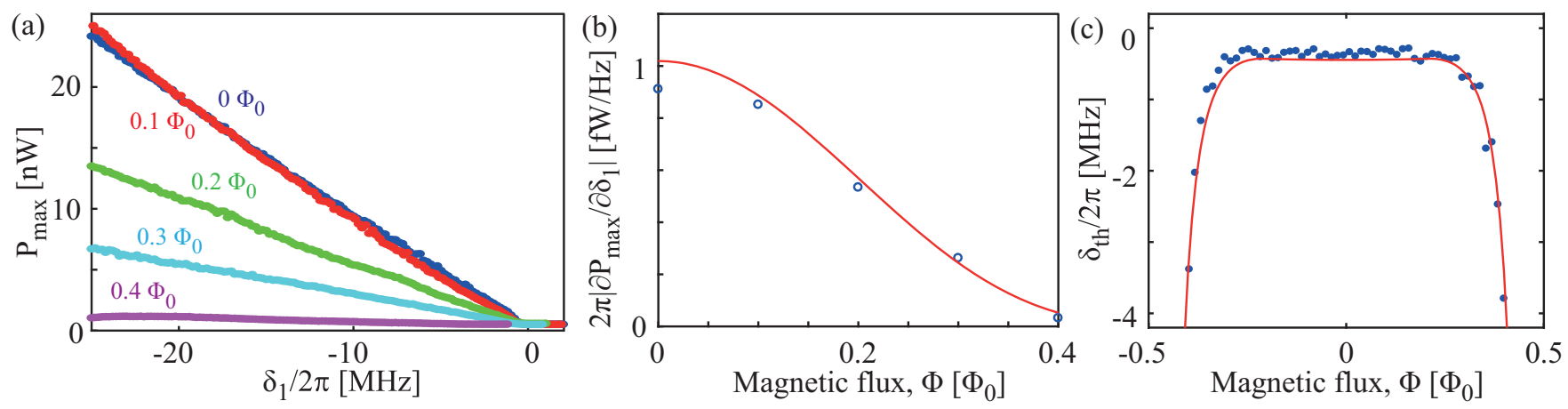

FIG. 6. (a) Growth of the intensity of subharmonic oscillations with red detuning for five different flux bias values. (b) Slopes of the data traces in (a) plotted versus magnetic flux (blue circles); the red line is the theory prediction [Eqs. (8) and (12)]. (c) Threshold detunings for subharmonic oscillations at different magnetic flux values; blue dots show experimental data, and the red line shows theory (see comment in the text).

resonator fundamental mode and in a finite interval of drive power. Due to the proximity of the second resonator mode to the drive tone, the down-conversion efficiency is strongly enhanced, enabling access to the subharmonic oscillation regime. A theory for the two-mode subharmonic resonance was developed to explain the observations. The theoretical predictions are in good quantitative agreement with the experimental observations regarding the boundary of existence of oscillations, maximum output power, and frequency threshold.

Our successful implementation of an intermode interaction of the $a_{1}^{\dagger 3} a_{2}$ type may in the future be used to create multiphoton entanglement and multicomponent macroscopic cat states [12].

\section{ACKNOWLEDGMENTS}

We thank W. Wustmann, M. Dykman, and G. Johansson for useful discussions. We gratefully acknowledge financial support from the European Research Council, the European project PROMISCE, the Swedish Research Council, and the Wallenberg Foundation. J.B. acknowledges partial support by the EU under REA Grant Agreement No. CIG-618353.

\section{APPENDIX}

In this appendix we derive quasiclassical solutions to Eq. (1), identify the stable solutions, and discuss the solution properties relevant for quantitative interpretation of the experimental data.

Before proceeding with solving Eq. (1), we reproduce the spectral equation for the tunable resonator [26,31] that is used for fitting the data in Fig. 3 and justifies the two-mode model for the resonator,

$$
\left(k_{n} d\right) \tan k_{n} d=\frac{2 E_{J}(\Phi)}{E_{L, \mathrm{cav}}}-\frac{2 C_{J}}{C_{\mathrm{cav}}}\left(k_{n} d\right)^{2} .
$$

Here, $k_{n}=\omega_{n} / v$ is the mode wave vector, $d$ is the length of the resonator, $E_{L \text {, cav }}$ is the inductive energy of the resonator, $C_{\text {cav }}$ is the resonator capacitance, $C_{J} \ll C_{\text {cav }}$ is the Josephson junction capacitance, and $2 E_{J}(\Phi)=2 E_{J} \cos \left(\pi \Phi / \Phi_{0}\right)$ is the Josephson energy of the SQUID.
It is useful to note that the quasiclassical version of the Hamiltonian (2), a metapotential, can be written in terms of quadratures, $\left[p_{n}=\operatorname{Re}\left(a_{n}\right), q_{n}=\operatorname{Im}\left(a_{n}\right)\right]$, of the form

$$
\begin{aligned}
H\left(p_{n}, q_{n}\right) / \hbar= & -\sum_{n}\left[\delta_{n}\left(p_{n}^{2}+q_{n}^{2}\right)+\alpha_{n}\left(p_{n}^{2}+q_{n}^{2}\right)^{2}\right] \\
& -2 \alpha\left(p_{1}^{2}+q_{1}^{2}\right)\left(p_{2}^{2}+q_{2}^{2}\right) \\
& +\frac{\tilde{\alpha}}{3}\left[q_{1} q_{2}\left(q_{1}^{2}-3 p_{1}^{2}\right)-p_{1} p_{2}\left(p_{1}^{2}-3 q_{1}^{2}\right)\right] .
\end{aligned}
$$

The phase portrait for the period-tripling subharmonic oscillator defined by this metapotential is presented in Fig. 7. It gives general information about the structure of the subharmonic oscillator stable steady states: they consist of four states, including the trivial ground state at the origin, $p_{1}=q_{1}=0$, and the three nontrivial states corresponding to the excited oscillator.

To establish the stability of the trivial solution to Eq. (1), $a_{1}=0$, we linearize this equation and assume time dependence

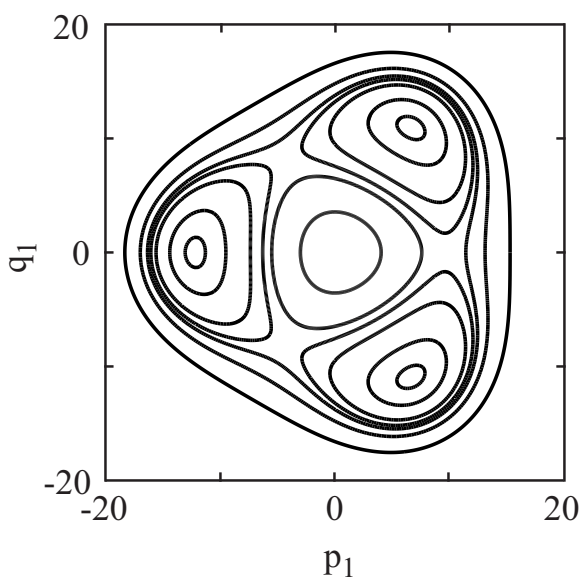

FIG. 7. Phase portrait for the subharmonic dynamics of the resonator fundamental mode defined by the metapotential, Eq. (A2), with fixed values $p_{2}$ and $q_{2}$ given by the experimental data point $\delta_{1} / 2 \pi=-12 \mathrm{MHz}$ and $\left|B_{2}\right|^{2}=6.25 \times 10^{10}$ photons $/ \mathrm{s}$. 

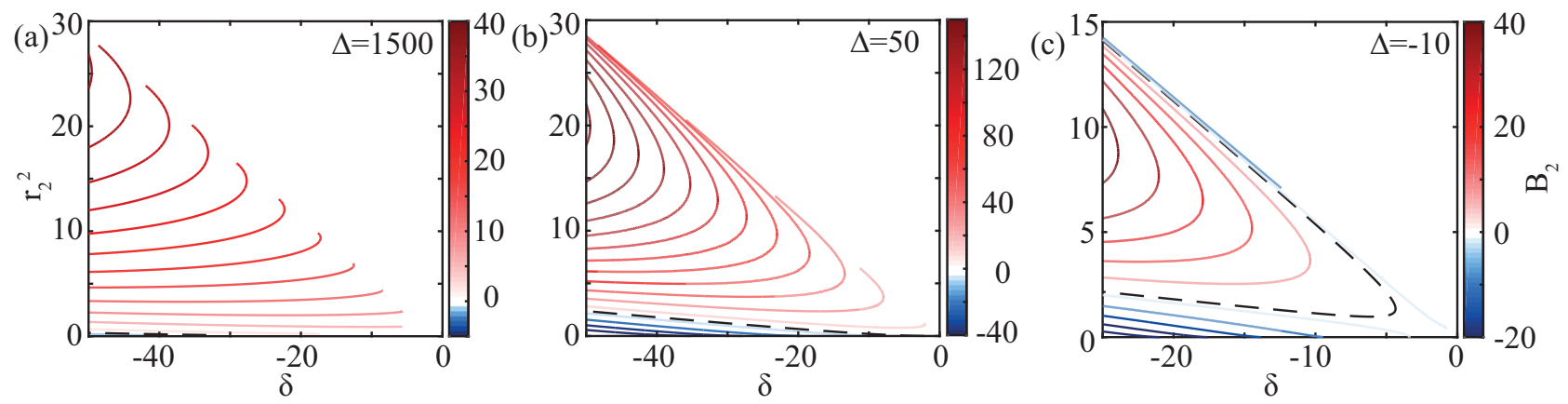

FIG. 8. Response of the second mode $r_{2}^{2}$ as a function of detuning for different drive amplitudes $B_{2}$ (represented by the color scale). The phase of the response is included in the sign of the drive amplitude. The panels illustrate the evolution of the response with decreasing spectrum anharmonicity. The curves are restricted to the region of existence of subharmonic oscillations. Exact resonance $\left(B_{2}=0\right)$ is indicated with a black dashed line. $\left(\gamma_{1}=1.93,0.10,0.08 ; \gamma_{2}=0\right.$. $)$

of the small fluctuation, $a_{1} \propto e^{\lambda_{0} t}$; then we find

$$
\lambda_{0}=i\left(\delta_{1}+2 \alpha\left|a_{2}\right|^{2}\right)-\Gamma_{1} .
$$

Since $\operatorname{Re} \lambda_{0}<0$, the trivial solution is always stable.

Solving Eq. (1) consists of two steps. First, a solution for the subharmonic oscillations of the first mode is constructed treating the field of the second mode as an effective pump $[13,14]$. Then the field of the second mode is computed as a nonlinear response to the drive. Analysis of Eq. (1) is convenient to perform using dimensionless parameters,

$$
\delta=\frac{\delta_{1}}{\alpha_{1}}, \Delta=\frac{3 \omega_{1}-\omega_{2}}{\alpha_{1}}, \frac{\delta_{2}}{\alpha_{1}}=3 \delta+\Delta, \gamma_{n}=\frac{\Gamma_{n}}{\alpha_{1}} .
$$

Derivations of the explicit equations for the external damping,

$$
\Gamma_{n, \mathrm{ext}}=\omega_{n}\left(k_{n} d\right)\left(\frac{C_{c}}{C_{\mathrm{cav}}}\right)^{2},
$$

where $C_{c}$ is the coupling capacitance, and for the Kerr coefficients,

$$
\alpha_{n}=\frac{\hbar \omega_{n}^{2} E_{L, \mathrm{cav}}^{2}}{16 E_{J}^{3}(\Phi)},
$$

are found in [26]. With these parameters and using the representation (3), the stationary equation (1) takes the form

$$
\begin{aligned}
& \quad\left(\delta+i \gamma_{1}+r_{1}^{2}+2 r_{2}^{2}\right) r_{1}+r_{2} r_{1}^{2} e^{-i\left(3 \phi_{1}-\phi_{2}\right)}=0, \\
& {\left[3 \delta+\Delta+i \gamma_{2}+\beta^{2}\left(r_{2}^{2}+2 r_{1}^{2}\right)\right] r_{2}+\frac{\beta^{2}}{3} r_{1}^{3} e^{i\left(3 \phi_{1}-\phi_{2}\right)}} \\
& =\beta \sqrt{\frac{2 \gamma_{2, \mathrm{ext}}}{\alpha_{1}}} B_{2} e^{-i \phi_{2}} .
\end{aligned}
$$

To solve Eq. (A7), we separate the real and imaginary parts,

$$
\begin{aligned}
\gamma_{1} & =r_{2} r_{1} \sin \left(3 \phi_{1}-\phi_{2}\right), \\
\delta+r_{1}^{2}+2 r_{2}^{2} & =-r_{2} r_{1} \cos \left(3 \phi_{1}-\phi_{2}\right),
\end{aligned}
$$

and eliminate the oscillator phase. Then we get a closed equation for $r_{1}$, which has the solutions

$$
r_{1}^{2}=-\left[\delta+(3 / 2) r_{2}^{2}\right] \pm \sqrt{-r_{2}^{2} \delta-(7 / 4) r_{2}^{4}-\gamma_{1}^{2}} .
$$

These solutions are restricted to the region defined by Eqs. (6) and (7). Equations for the phase $\phi_{1}$, extracted from Eq. (A9), read

$$
\begin{aligned}
& \sin \left(3 \phi_{1}-\phi_{2}\right)=\frac{\gamma_{1}}{r_{2} r_{1}}>0, \\
& \cos \left(3 \phi_{1}-\phi_{2}\right)= \pm \sqrt{1-\frac{\gamma_{1}^{2}}{\left(r_{2} r_{1}\right)^{2}}}=\frac{-\delta-2 r_{2}^{2}-r_{1}^{2}}{r_{2} r_{1}} .
\end{aligned}
$$

The solutions have a threefold degeneracy: for every given value of the phase $\phi_{2}$, there are three values of the subharmonic oscillation phase $\phi_{1}$ shifted by $2 \pi / 3 \mathrm{rad}$ with respect to each other (see Fig. 7).

To evaluate the stability of these solutions, we use their simplified forms for brevity, which is valid away from the threshold, $|\delta| \gg \gamma_{1}$,

$$
3 \phi_{1}-\phi_{2}=0, \pi, r_{1}=\mp\left(\frac{r_{2}}{2} \pm \sqrt{|\delta|-\frac{7}{4} r_{2}^{2}}\right)
$$

(the minus and plus signs in front of the parentheses correspond to the zero and $\pi$ phase differences, respectively). The linearized equation for the small fluctuation $\delta a_{1}$ around each of the steady-state solutions has the form

$$
i \delta \dot{a}_{1}+\left(\delta+2 r_{1}^{2}+2 r_{2}^{2}\right) \delta a_{1}-\left(r_{1}^{2}+2 \delta+4 r_{2}^{2}\right) \delta a_{1}^{*}=0 .
$$

Assuming $\operatorname{Re}\left(\delta a_{1}\right), \operatorname{Im}\left(\delta a_{1}\right) \propto e^{\lambda_{1} \alpha_{1} t}$, for this solution we find from Eqs. (A13) and (A12)

$$
\lambda_{1}^{2}= \pm 6 r_{1}^{2} r_{2}\left(r_{1} \pm r_{2} / 2\right) .
$$

For the minus sign in front of the parentheses, which corresponds to $3 \phi_{1}-\phi_{2}=\pi$ in Eq. (A12), the exponent is

$$
\lambda_{1}^{2}=-6 r_{2}^{2}\left( \pm \sqrt{|\delta|-\frac{7}{4} r_{2}^{2}}\right) .
$$

For the positive root, $\lambda_{1}^{2}<0$; hence the solution with both signs positive in Eq. (A12) is stable. The full form of this solution is presented in the main text in Eqs. (4) and (5). The other choices of the signs result in positive $\lambda_{1}>0$, hence corresponding to unstable solutions.

Equation (A8) describes a Duffing oscillator perturbed by the back-action of the subharmonic oscillator. The imaginary part of this equation defines the phase of the response $\phi_{2}$. 


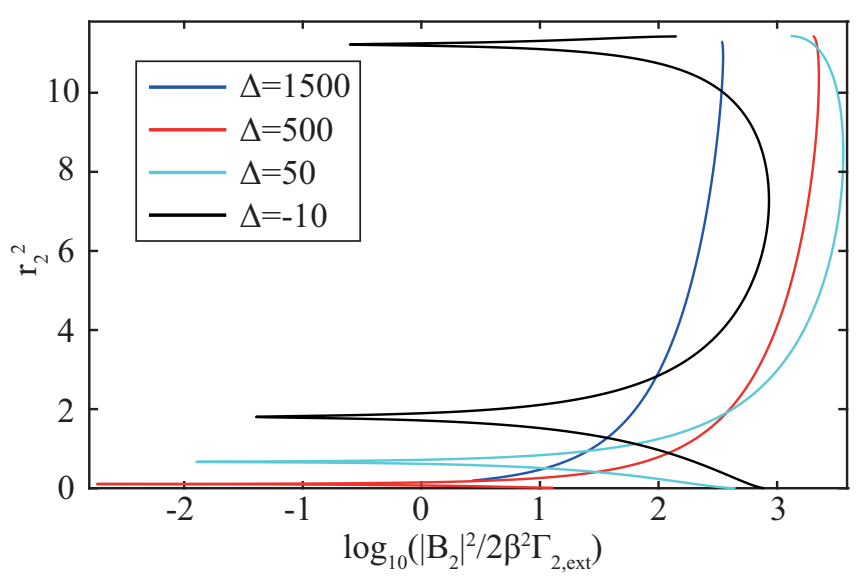

FIG. 9. Response of the second mode $r_{2}^{2}$ as a function of drive power for different values of the spectrum anharmonicity, $\Delta=1500,500,50,-10$, at $\delta=-20$, corresponding to $\Phi=0.1,0.32,0.406,0.4178 \Phi_{0}\left(\gamma_{1}=1.93,0.43,0.10,0.08, \gamma_{2}=\right.$ $\left.13.6,2.94,0.71,0.55, \gamma_{2, \text { ext }}=13.1,2.84,0.69,0.53\right)$.

Similar to Eq. (A11), the difference between this phase and the phase of the drive $\phi_{B}$ is defined by the damping $\gamma_{2}$, and for the major parameter interval of interest, $\Delta,|\delta| \gg \gamma_{2}$, phase $\phi_{2}$ is either close to the phase of the drive or shifted by $\pi$, $\phi_{2}-\phi_{B} \approx 0, \pi$ [compare Eq. (A12)]. The amplitude of the response is found from the equation

$$
\begin{aligned}
& \left\{\left[3 \delta+\Delta+\beta^{2}\left(r_{2}^{2}+2 r_{1}^{2}\right)\right] r_{2}-\frac{\beta^{2}}{3} r_{1}^{3}\right\}^{2}+\gamma_{2}^{2} r_{2}^{2} \\
& \quad=\beta^{2} \frac{2 \gamma_{2, \text { ext }}}{\alpha_{1}}\left|B_{2}\right|^{2} .
\end{aligned}
$$

The dependence $r_{2}^{2}(\delta)$ for different drive amplitudes $B_{2}$ and flux values is illustrated in Fig. 8. For better clarity the plots are made neglecting damping of the second mode on the left-hand side and including the phase of the response in the sign of the drive amplitude; then positive $B_{2}>0$ correspond to $\phi_{2}=\phi_{B}$, and negative $B_{2}<0$ correspond to $\phi_{2}=\phi_{B}+\pi$. The response qualitatively resembles the one of the Duffing oscillator; the similarity is most pronounced at small values of the spectrum anharmonicity illustrated in Figs. 8(b) and 8(c) for $\Delta=50$ and $\Delta=-10$. Here, the bistability region is seen at $B_{2}>0$ as well as the exact resonance, $B_{2}=0$, which is indicated with a black dashed line in Fig. 8(c). The stable solutions correspond to the lower branch at positive $B_{2}$ and the branch with negative $B_{2}$ above the resonance. There is, however, a second resonance that appears at smaller values of $r_{2}$; the states below this resonance line, at negative $B_{2}$, are unstable.

The dependence $r_{2}^{2}\left(|B|^{2}\right)$ from Eq. (A8) is illustrated in Fig. 9 for a representative value of the detuning, $\delta=-20$, and for different values of the spectrum anharmonicity $\Delta$, which is controlled by the bias magnetic flux $\Phi$. When the spectrum anharmonicity is large, $\Delta=1500,500\left(\Phi=0.1,0.32 \Phi_{0}\right)$, the stable solution for $r_{2}$ exists for all drive amplitudes except for very small values, where the second, unstable solution appears (this solution corresponds to the region below the second resonance in Fig. 8). In this region of large anharmonicity, which significantly exceeds the experimental interval of detunings, Eq. (A8) can be significantly simplified by dropping $|\delta| \ll \Delta, r_{1}^{2}, r_{2}^{2} \lesssim|\delta|$,

$$
\Delta^{2} r_{2}^{2}=\frac{2 \beta^{2} \gamma_{2, \mathrm{ext}}}{\alpha_{1}}\left|B_{2}\right|^{2}
$$

Inserting Eq. (8) into this equation, we obtain the maximum drive power at which the subharmonic oscillations may persist,

$$
\left|B_{2}\right|^{2} \approx \frac{2 \Delta^{2}|\delta| \alpha_{1}}{7 \beta^{2} \gamma_{2, \mathrm{ext}}}, \quad|\delta| \gg \gamma_{1} .
$$

When the anharmonicity decreases $(\Delta \lesssim 50, \Phi \approx 0.41)$, an unstable (back-bending) branch emerges at large drive. This feature is associated with the bifurcation in Fig. 8. This effect should lead to a reduction of the visible part of the subharmonic oscillation region in Fig. 4(a). With a further decrease of the anharmonicity the subharmonic oscillations should disappear at $\Phi \gtrsim 0.4 \Phi_{0}$.
[1] S. H. Strogatz, Nonlinear Dynamics and Chaos (Westwood Press, Boulder, Colorado, 2015).

[2] M. C. Cross and P. C. Hohenberg, Rev. Mod. Phys. 65, 851 (1993).

[3] R. Z. Sagdeev, D. A. Usikov, and G. M. Zaslavsky, Nonlinear Physics: From the Pendulum to Turbulence and Chaos (Harwood Academic Publishers, Chur, Switzerland, 1988).

[4] I. Siddiqi, R. Vijay, F. Pierre, C. M. Wilson, M. Metcalfe, C. Rigetti, L. Frunzio, and M. H. Devoret, Phys. Rev. Lett. 93, 207002 (2004).

[5] Z. R. Lin, K. Inomata, K. Koshino, W. D. Oliver, Y. Nakamura, J. S. Tsai, and T. Yamamoto, Nat. Commun. 5, 4480 (2014).

[6] P. Krantz, A. Bengtsson, M. Simoen, S. Gustavsson, V. Shumeiko, W. D. Oliver, C. M. Wilson, P. Delsing, and J. Bylander, Nat. Commun. 7, 11417 (2016).
[7] C. Eichler, D. Bozyigit, C. Lang, M. Baur, L. Steffen, J. M. Fink, S. Filipp, and A. Wallraff, Phys. Rev. Lett. 107, 113601 (2011).

[8] N. Bergeal, F. Schackert, L. Frunzio, and M. H. Devoret, Phys. Rev. Lett. 108, 123902 (2012).

[9] E. Flurin, N. Roch, F. Mallet, M. H. Devoret, and B. Huard, Phys. Rev. Lett. 109, 183901 (2012).

[10] C. Macklin, K. O’Brien, D. Hover, M. E. Schwartz, V. Bolkhovsky, X. Zhang, W. D. Oliver, and I. Siddiqi, Science 350, 307 (2015).

[11] M. Mirrahimi, Z. Leghtas, V. V. Albert, S. Touzard, R. J. Schoelkopf, L. Jiang, and M. H. Devoret, New J. Phys. 16, 045014 (2014).

[12] S. Puri, S. Boutin, and A. Blais, npj Quantum Inf. 3, 18 (2017).

[13] C. Hayashi, Nonlinear Oscillations in Physical Systems (Princeton University Press, Princeton, NJ, 1985). 
[14] D. W. Jordan and P. Smith, Nonlinear Ordinary Differential Equations (Oxford University Press, Oxford, 2007).

[15] H. Haken, Rev. Mod. Phys. 47, 67 (1975).

[16] Y. Zhang, J. Gosner, S. M. Girvin, J. Ankerhold, and M. I. Dykman, arXiv:1702.07931.

[17] C. F. Spitzer, J. Appl. Phys. 16, 105 (1945).

[18] P. S. Linsay, Phys. Rev. Lett. 47, 1349 (1981).

[19] W. F. Ngai and H. Liu, Appl. Phys. Lett. 62, 2611 (1993).

[20] J. M. Soto-Crespo, M. Grapinet, P. Grelu, and N. Akhmediev, Phys. Rev. E 70, 066612 (2004).

[21] B. A. Huberman, J. P. Crutchfield, and N. H. Packard, Appl. Phys. Lett. 37, 750 (1980).

[22] R. L. Kautz, J. Appl. Phys. 52, 6241 (1981).

[23] I. Siddiqi, R. Vijay, F. Pierre, C. M. Wilson, L. Frunzio, M. Metcalfe, C. Rigetti, R. J. Schoelkopf, M. H. Devoret, D. Vion, and D. Esteve, Phys. Rev. Lett. 94, 027005 (2005).

[24] R. Vijay, M. Devoret, and I. Siddiqi, Rev. Sci. Instrum. 80, 111101 (2009).

[25] C. M. Wilson, T. Duty, M. Sandberg, F. Persson, V. Shumeiko, and P. Delsing, Phys. Rev. Lett. 105, 233907 (2010).
[26] W. Wustmann and V. Shumeiko, Phys. Rev. B 87, 184501 (2013).

[27] T. Yamamoto, K. Koshino, and Y. Nakamura, in Principles and Methods of Quantum Information Technologies, edited by Y. Yamamoto and K. Semba (Springer, Tokyo, 2016), pp. 495-513.

[28] L. Guo, M. Marthaler, and G. Schön, Phys. Rev. Lett. 111, 205303 (2013)

[29] M. Denisenko, V. Munyayev, and A. Satanin, J. Phys. Conf. Ser. 681, 012018 (2016).

[30] M. Sandberg, C. M. Wilson, F. Persson, T. Bauch, G. Johansson, V. Schumeiko, T. Duty, and P. Delsing, Appl. Phys. Lett. 92, 203501 (2008).

[31] M. Wallquist, V. S. Shumeiko, and G. Wendin, Phys. Rev. B 74, 224506 (2006).

[32] A. Palacios-Laloy, F. Nguyen, F. Mallet, P. Bertet, D. Vion, and D. Esteve, J. Low Temp. Phys. 151, 1034 (2008).

[33] E. Zakka-Bajjani, F. Nguyen, M. Lee, L. R. Vale, R. W. Simmonds, and J. Aumentado, Nat. Phys. 7, 599 (2011).

[34] W. Wustmann and V. Shumeiko, Phys. Rev. Appl. 8, 024018 (2017). 